\title{
Distributed Norm Enforcement via Ostracism
}

\author{
Adrian Perreau de Pinninck, Carles Sierra, and Marco Schorlemmer \\ IIIA - Artificial Intelligence Research Institute \\ CSIC - Spanish National Research Council \\ Bellaterra (Barcelona), Catalonia, Spain \\ adrianp,sierra,marco@iiia.csic.es
}

\begin{abstract}
An agent normative society has to deal with two main concerns: how to define norms and how to enforce them. Enforcement becomes a complex issue as agent societies become more decentralized and open. We propose a new distributed mechanism to enforce norms by ostracizing agents that do not abide by them. Our simulations have shown that, although complete ostracism is not always possible, the mechanism substantially reduces the number of norm violations.
\end{abstract}

\section{Introduction}

In a normative Multi-Agent System (MAS) a set of norms are added to restrict the set of available actions in order to improve the coordination between agents. An autonomous agent has the choice whether or not to support a norm. It is up to the agent to decide if it is convenient for it to abide by it. For a utility maximizer agent if following a norm is profitable, it is in the agent's own interest to act as the norm establishes. But this is not always the case, as some norms are profitable even when not all agents abide by them. For example, a norm that dictates that owners must clean the common areas. Cleaning entails a cost, and a clean area is a benefit to all. If an owner does not clean the common area (i.e., a norm violator) thus not bearing its cost, but the others do, the area is still clean.

The aim of this paper is to introduce a new distributed mechanism that attains norm compliance by ostracizing norm violating agents. Our scenario allows agents to interact with each other. An agent can interact with the agents it is linked to directly or indirectly through a path of links (i.e., agents can interact with direct neighbors, with neighbors of neighbors, and with their neighbors and so on...). An initiator agent will search for a path in the society to find a partner agent with which to interact. All the agents in the path that are not the initiator or the partner agent will be called mediator agents (i.e., agents mediating the interaction).

We use a game-theoretic approach to interactions, which we model as a twoplayer game with two possible strategies; cooperate and defect. The utility function will be that of a prisoner's dilemma (see Figure 1).

The norm in this scenario is for all agents to cooperate, thus attaining the maximum utility for the society. Nonetheless, agents can choose to ignore the 


\begin{tabular}{|c||c|c|}
\hline PD & Cooperate & Defect \\
\hline \hline Cooperate & 3,3 & 0,5 \\
\hline Defect & 5,0 & 1,1 \\
\hline
\end{tabular}

Fig. 1. Prisoner's Dilemma Payoff Matrix

norm and defect (i.e., violate the norm) thus gaining more utility. In order to attain norm enforcement, some agents (we will call them enforcer agents) are given the ability to stop interacting with violators, and to stop them from interacting with the enforcer's own neighbors. When enough agents use this ability against a violator, it is ostracized. An agent is ostracized when it cannot interact with anyone else in the society, in this case it is a consequence of defecting in the interaction against many different agents.

The motivation behind using ostracism comes from the study of norm enforcement in primitive societies [11]. When a member of a community repeatedly ignored its customs, it was forced to leave. No one in the community would interact with the ostracized member from then on. Ostracism is achieved in human societies through force and physical constraint. In order to achieve ostracism of electronic entities, which interact through a network, we seek inspiration from the network security area. The most commonly used component in this case is a firewall, which blocks those communications which appear to be harmful. While firewalls are usually set up by humans through complex rules, enforcer agents will use gossip as a way to inform each other about malicious agents.

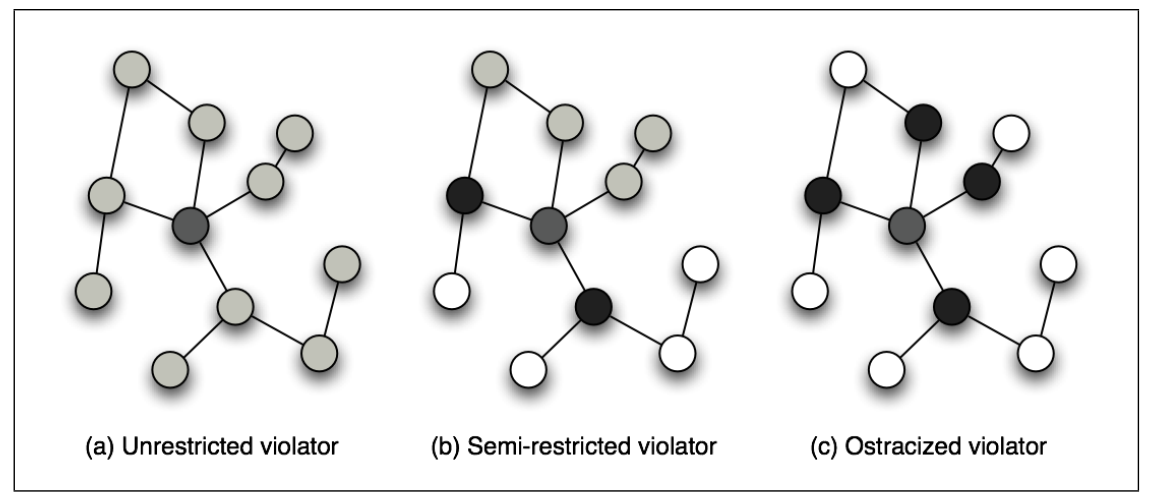

Fig. 2. Ostracizing a violator

The ostracism process can be seen in Figure 2. At first an undetected violator in the network (the dark gray node) can interact with all the other agents (light gray nodes are liable to interact with the violator). When the violator interacts, and defects, it can be detected by enforcer agents which will block it (black 
nodes are blocking agents, and white nodes are agents that the violator cannot interact with). When all the violator's neighbors block it, it is ostracized.

Gossip is essential to find out information about other agents in a distributed environment. We will use gossip as part of the enforcement strategy to ostracize agents. Information is gossiped only to agents mediating the interaction, to minimize the amount or resources it takes. If agent $a g_{v}$ violates the norm when interacting with agent $a g_{1}, a g_{1}$ may spread this information to all mediator agents so they may block $a g_{v}$ in the future.

By running a set of simulations, we study under which conditions the mechanism works, and give measures of its success (such as the violations received or the utility gained). Our hypotheses are:

- H1 - Norm violations can be reduced by applying a local blocking rule.

- H2 - The society's structure influences its enforcement capabilities.

- H3 - The choice of blocking strategy influences the number of violations received.

- H4 - Enforcement makes norm-abiding a rational strategy.

Section 2 describes related work in the area of norm enforcement. Section 3 presents a detailed description of the scenario we employ in the simulations. Section 4 describes the simulations and analyzes the resulting data. Finally, section 5 presents future work.

\section{Related Work}

The problem of norm enforcement has been dealt with in human societies through the study of law, philosophy, and the social sciences. Recently it is being dealt with in computer science, where norms are studied as a coordination mechanism for multi-agent systems. Axelrod [1] first dealt with the application of norms from an evolutionary perspective. Enforcement is seen by Axelrod as a sort of meta-norm to punish agents that do not punish violators. The norm game is often modeled as an N-Player Iterated Prisoner's Dilemma [1, 8]. In these cases the norm is to cooperate and ways are sought to ensure that agents prefer cooperation. Other research studies norms that avoid aggression or theft $[4,7,12,15]$. In these cases agents gain utility by either finding items or receiving them as gifts. But these items can be stolen by other agents through aggression. An agent that abides by the possession norms will not steal food possessed by another agent, therefore avoiding aggression.

Two enforcement strategies have been studied to attain norm compliance: the use of power to change the utilities through sanctions or rewards $[2,3,8$, 14], and the spread of normative reputation in order to avoid interaction with violators $[4,6,7,12,15]$. Both strategies have the goal of making norm adopters better off than norm violators. But this is not always accomplished $[4,7]$, since all agents benefit from the norm while only enforcers agents bear its cost.

Norm enforcement models in $[2,6]$ show how violating the norm becomes an irrational strategy when punishment is possible. But these models assume the 
following: (i) agents are able to monitor other agents' activities; and (ii) agents have the ability to influence the resulting utility of interactions. Assumption (i) can be materialized by having a central agent mediate all interactions [2], or by having agents recognize violators through direct interaction with them, or through gossip with other agents [4]. The first solution does not scale, since the mediator agent would be overwhelmed in a large system. The second scales because no agent is the enforcement bottleneck, but it is less efficient since in a distributed environment not all violations can be detected. Assumption (ii) can be carried out through third-party enforcement [2], or self-enforcement [6] in which each agent carries out sanctions to agents it interacts with. Third party does not scale since it can easily be overwhelmed in a large system. For selfenforcement, all agents must have the ability to affect the outcome utility of interactions.

Axelrod [1] defines the "shadow of the future" as a mechanism to affect an agent's choice in iterated games. An agent is deterred from defecting when the probability of interacting with the same agent in the future is high, and agents will defect in future interactions with known violators. Nonetheless, this mechanism makes enforcers violate the norm as they also defect. Another method is the threat of ostracism or physical constraint. By not interacting with violators, an agent can interact with another agent and achieve a higher payoff. Younger has studied [15] the possibility of avoiding interaction with norm-violators, but does not prevent norm-violators from interacting with anyone else.

Kittock [9] was the first to study how the structure of a multi agent system affected the emergence of a social norm. He studied regular graphs, hierarchies, and trees. In [5] Delgado studied emergence in complex graphs such as scale-free and small-world, and in [10] studied the relationship between a graph's clustering factor and emergence.

Using the scenario presented in this paper, agents can monitor other agents' activities, and influence future interactions. The spread gossip, and sanctioning norm-violators with ostracism via blockage are the techniques used to achieve this influence. We have studied norm enforcement using these techniques in societies with differing structures.

\section{The Scenario}

We model our multi-agent system as an undirected, irreflexive graph: $M A S=$ $\langle A g, R e l\rangle$, where $A g$ is the set of vertices and $R e l$ the set of edges. Each vertex models an agent and each edge between two vertices denotes that the agents are linked to each other. We have chosen three kinds of graphs for their significance: Tree, Random, and Small-World. We define a tree as a graph in which each node has one parent and some number of children; one node, the root node, has no parent, and the leave nodes have no children. Nodes are linked to their parents and children. In a random graph any node can be linked to any other one with a given probability. A small-world graph is created by starting with a 
regular graph ${ }^{1}$, and adding enough random edges to make the average distance between any two vertices significantly smaller [13]. A small-world graph is highly clustered (i.e., if a node has two neighbors, the probability of them being linked is high), and there are some links between distant parts of the graph that make the average distance between any two edges small. The graph structures have been generated with a similar average number of links per node.

We use a game-theoretic approach by modeling interactions as a two-player prisoner's dilemma game. The norm is that agents ought to cooperate (i.e., an agent disobeys the norm by defecting). In order for two agents to interact, there must be a path in the graph between the two. One agent will search for a path that leads to another agent with which to interact. We call the searching agent initiator agent, the agent chosen to interact partner agent, and the remaining agents in the path mediator agents. The partner finding process is explained below, but first we need to formally describe some terms.

We define the set of neighbors of an agent $a_{i}$ as the set of agents it is linked to directly in the graph: $N\left(a_{i}\right)=\left\{a_{j} \in A g \mid\left(a_{i}, a_{j}\right) \in\right.$ Rel $\}$. Each agent also has a set of agents it blocks (an agent cannot block itself ): $B\left(a_{i}\right) \subseteq A g \backslash\left\{a_{i}\right\}$. An agent $a_{i}$ can query another agent $a_{j}$ for a list of its neighbors. We call the set of agents that $a_{j}$ returns, reported neighbors: $R N\left(a_{i}, a_{j}\right) \subseteq N\left(a_{j}\right)$. The set of reported neighbors depends on the blocking strategy of $a_{j}$. The strategies used in our simulations are explained below. A path is the route (without cycles) in the graph structure through which interaction messages are delivered. We represent a path as a finite (ordered) sequence of agents $p=\left[a_{1}, a_{2}, \ldots, a_{n}\right]$ such that for all $i$ with $1 \leq i \leq n-1$ and $n \geq 2$ we have that $a_{i+1} \in N\left(a_{i}\right)$, and for all $i, j$ with $1 \leq i, j \leq n$ and $i \neq j$ we have that $a_{i} \neq a_{j}$. The agent $a_{1}$ of a path is the initiator agent, agent $a_{n}$ is the partner agent, the remaining ones are mediator agents.

In order to find a partner, the initiator agent $a_{i}$ creates a path $p=\left[a_{i}\right]$ with itself as the only agent in it. A path with one agent is not valid, since an agent cannot interact with itself. Therefore, the initiator agent will query the last agent in the path (the first time it will be itself) to give it a list of its neighbors. It will choose one of them randomly ${ }^{2}\left(a_{j}\right)$ and add it to the end of the path $p=\left[a_{i}, \ldots, a_{j}\right]$. At this point, if agent $a_{j}$ allows it, the initiator agent can choose agent $a_{j}$ as the partner. Otherwise, it can query agent $a_{j}$ for its neighbors and continue searching for a partner. This choice is taken randomly, with probability $p=0.3 a_{j}$ becomes the partner, and with probability $1-p$ it becomes a mediator and $a_{i}$ asks it for its neighbors.

If the path's last element is an agent $a_{n}$ that refuses to interact with the initiator agent, and $a_{n}$ returns an empty list of agents when queried for its neighbors, backtracking is applied. Agent $a_{n}$ is removed and a different agent is chosen from the list of $a_{n-1}$ 's neighbors and added to the end of the list.

\footnotetext{
${ }^{1} C_{N, r}$ is a regular graph on $N$ vertices such that vertex $i$ is adjacent to vertices $(i+j) \bmod N$ and $(i-j) \bmod N$ for $1 \leq j \leq r$.

${ }^{2}$ To avoid loops, an agent that is already part of the path cannot be chosen again.
} 
Once the partner is chosen, a prisoner's dilemma game is played between the initiator and the partner. The game results and the path are known by both playing agents. Playing agents can choose to send the game results to all the mediators in the path. This is what we call gossip, which formally speaking is a tuple that contains the agents' names and their strategy choices for the given game: Gossip $=\left\langle a g_{i}, c h_{i}, a g_{j}, c h_{j}\right\rangle$, where $c h_{i}$ and $c h_{j}$ are either cooperate or defect.

During the whole process agents can execute any of the following actions:

- Return a list of neighboring agents when asked for its neighbors.

- Accept, or reject, an offer to interact.

- Choose a strategy to play in the PD game when interacting.

- Inform mediators of the outcome of the interaction.

The society of agents is composed of three types of agents, each one characterized by a different strategy for the actions it can execute. A meek agent is a norm-abiding agent that always cooperates. It will always return all its neighbors to any agent that asks. A meek agent will always accept an offer to interact, it will always cooperate in the PD game, and it will never gossip. A violator agent follows the strategy of a meek agent, except that it always defects when playing a game, therefore it is not a norm-abiding agent. Violator agents in our simulations are very naive, they never model the other agents, or treat them differently depending on their actions. In short, they cannot change the strategies. Future work will look into more sofisticated norm-violators.

Finally, an enforcer agent has the ability to block violators, which is essential in order to achieve their ostracism. An enforcer agent shares the same strategies with meek agents with the following exceptions: It will add agents that have defected against it to its set of blocked agents, and will gossip to all mediators when defections happen. If an enforcer is informed of the results of a game it was mediating, it will act as if it had played the game itself. An enforcer agent will never choose an agent in its blocked set as a partner, and will not allow an agent in its blocked set to choose it as a partner. Therefore, a violator agent cannot interact with an enforcer who is blocking it. When an enforcer agent $a_{m}$ is asked to return a list of its neighbors by an agent $a_{i}$ who is not in its blocked set, two different strategies are possible. The Uni-Directional Blockage (UDB) strategy, where all its neighbors will be returned $\left(R N\left(a_{i}, a_{m}\right)=N\left(a_{m}\right)\right)$. Or the Bi-Directional Blockage (BDB) strategy, where only those neighbors not in its blocked set are returned $\left(R N\left(a_{i}, a_{m}\right)=N\left(a_{m}\right) \backslash B\left(a_{m}\right)\right)$. When the querying agent is in the enforcer agent's blocked set, it always returns an empty set.

The choice of enforcement strategy entails a trade off. Intuitively, one can see that enforcer agents are better off with the UDB strategy, since they will be able to use violator agents as mediators to reach other parts of the society. Enforcers will not be tricked by a violator more than once, so they are sure not to interact with them. Therefore, using violators as mediators benefits enforcers. Meek agents, on the other hand, do not learn to avoid violators. They may choose one unknowingly as their partner repeatedly. BDB is a better strategy for meek agents, it reduces their chances of choosing violator agents. Furthermore, a 
structure with a violator as a cut vertex, may be split into two different societies when the BDB strategy is used, and the violator is ostracized. If the UDB strategy is used, the society stays connected, since the ostracized violator can stil be used as a mediator.

In order to focus on the most relevant aspects in our simulations, we made the following limiting assumptions:

- Agents cannot change their strategy (i.e., a violator is always a violator).

- Agents cannot lie when sending gossip.

- There are no corrupt enforcer agents.

- There is no noise (i.e., an agent knows its opponent's chosen strategy).

These assumptions imply that modeling agents' reputation is simple. Being informed once about an agent's strategy is enough, since information will never be contradictory. Therefore, there is no place for forgiveness, and sanctions are indefinite. Relaxation of these assumptions will be studied in future work.

\section{Simulations}

The simulations have been run using the scenario specified in Section 3. Each simulation consists of a society of 100 agents. The society will go through 1000 rounds, in a round each agent tries to find a partner with which to interact. If the agent finds a partner a prisoner's dilemma with the utility function of Figure 1 is played.

The parameters that can be set in each simulation are:

- Percentage of Violators (V) - from $10 \%$ to $90 \%$ in $10 \%$ increments.

- Percentage of Enforcers (E) - from 0\% to $100 \%$ in $10 \%$ increments $^{3}$.

- Type of Graph (G) - either tree, small world, or random.

- Enforcement Type (ET) - Uni-Directional Blockage (UDB), or Bi-Directional Blockage (BDB).

An exhaustive set of simulations have been run with all the possible values for each parameter. Each simulation has been run 50 times in order to obtain an accurate average value. The metrics that have been extracted are: the mean violations received per agent, and the mean utility gained per agent. The metrics have been calculated for the whole society and for each agent type. The data gathered from the simulations supports our hypotheses.

(H1) Norm violations can be reduced by applying a local blocking rule. The graph in Figure 3(a) shows that the higher the percentage of normabiding agents that use a blocking rule, the lower the average number of norm violations received by any agent in our system. There are five different lines in the graph, each one stands for a different percentage of violating agents. In all cases a higher enforcer to meek agent ratio ( $x$-axes) leads to lower violations

${ }^{3}$ The percentage of meek agents is computed through the following formula: $M=$ $100 \%-V-E$. Therefore, $V+E$ cannot be more than $100 \%$. 
received in average by any agent ( $y$-axes). When the ratio of enforcers is high, violators end up interacting with each other. Therefore, the $y$-axes measures the violations received by "any" agent, the reduction in violations in Figure 3(a) is not significant. The data referring to the violations received only by normabiding agents shows a larger reduction (see Figure 3(b)). Enforcer agents can perceive a norm violation at most once per violator agent. But if we look at the violations received by meek agents, we see that they experience an increment of violations when the ratio of enforcers is high (see Figure 7(a)). This means that enforcer agents have blocked violator agents, which are forced to interact with the small number of meek agents left unprotected. Since the meek are a small portion of the norm supporters, this does not influence the total violations perceived by norm supporters as a whole. Therefore, the higher the ratio of enforcer agents, the lower the average of violations perceived by norm-abiding agents.

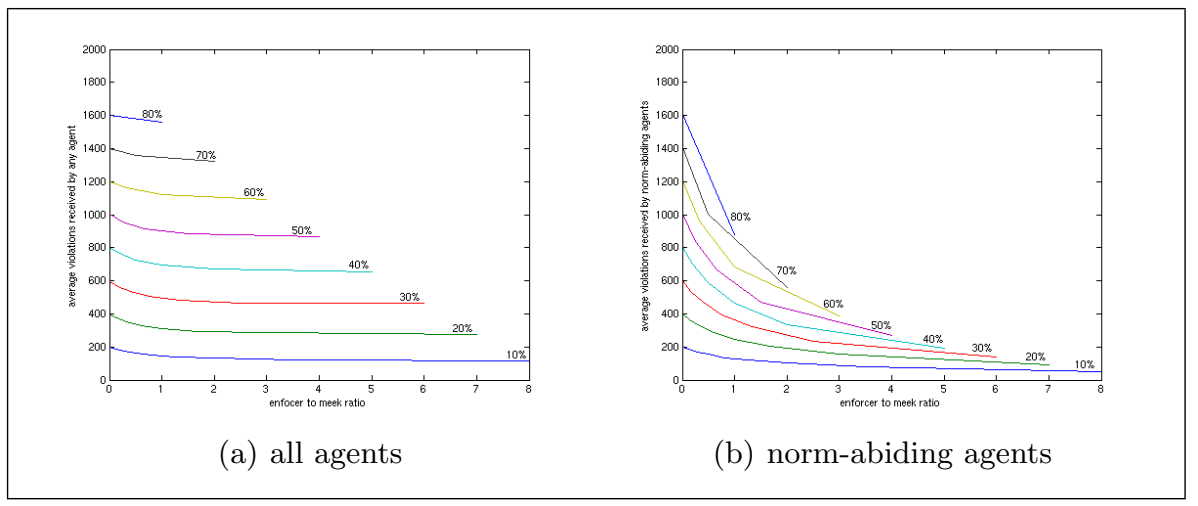

Fig. 3. Local blocking rule reduces violations

(H2) The society's structure influences its enforcement capabilities. It is also seen from the data that different organizational structures in the multi-agent system influence norm enforcement. In Figure 4(a) and 4(b) we have extracted the average norm violations ( $y$-axes) for each of the different structures tested: Random, Small World, and Tree. We have only shown the simulations where violator agents account for $10 \%$ and $20 \%$ of the population, therefore at most there will be $90 \%$ or $80 \%$ of enforcers, respectively. The $x$-axes contains the different percentages of enforcer agents tested. It can be seen that both random and small world networks have an almost identical graph line. On the other hand the tree structure has shown to improve the enforcement capabilities. The main difference between a tree and the other structures studied is that in a tree there is only one path between any two agents. In random and small world graphs, many paths can be usually found between any two agents.

(H3) The choice of blocking strategy influences the number of violations received. The data in Figure 5 supports this hypothesis. The $x$-axes shows 


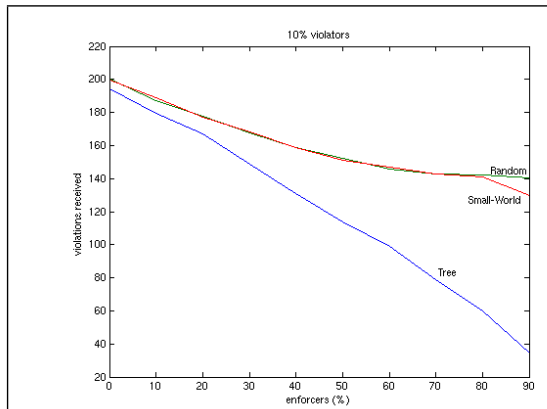

(a) $10 \%$ violators

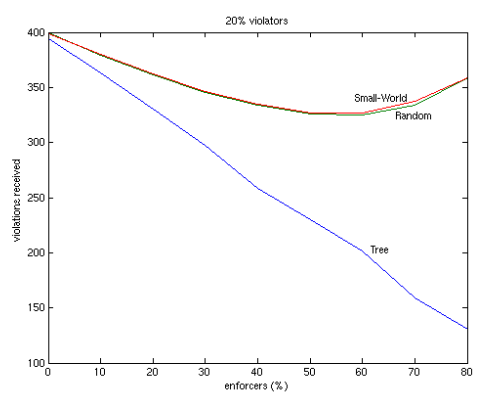

(b) $20 \%$ violators

Fig. 4. Enforcement capabilities vary depending on structure

the enforcer to meek agent ratio. The $y$-axes contains a metric for the increment in efficiency at protecting meek agents from violations. Efficiency is the difference (calculated in percentage) in violations received by meek agents for each of the two different enforcement strategies $\Delta E=\left(\left(V_{U D B} / V_{B D B}\right)-1\right) \times 100 . \Delta E$ calculates the increase in violations received by agents when using uni-directional blockage in respect to bi-directional blockage.

Figure 5 shows that for random and small world networks the efficiency is positively correlated with the enforcer to meek agent ratio. We can conclude that Bi-Directional Blockage has a higher efficiency at protecting meek agents from violator agents. This is not observed in the tree network. In this case the efficiency stays along the $0 \%$ line with some deviations. We argue that in networks organized as trees, the choice of enforcement strategy does not have a significant influence in the outcome. The tree network is already good for ostracizing offenders, and the blockage strategy does not improve it.

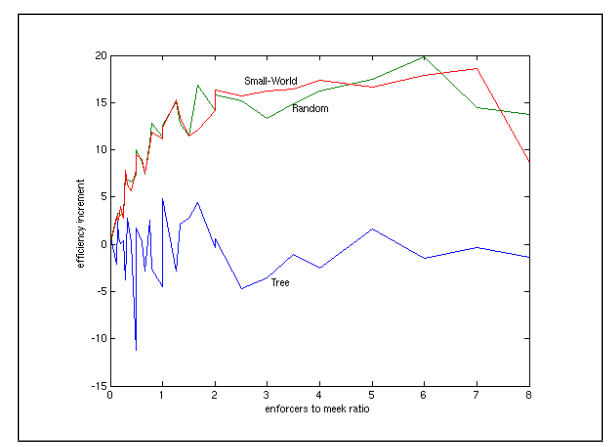

Fig. 5. Enforcement strategy influences received violations 
(H4) Enforcement makes norm-abiding a rational strategy. This hypothesis is supported by the utility gained by agents. A strategy is rational if it maximizes the agent's utility. What has been tested is whether following the norm maximizes the agent's utility, and in which conditions. Figure 6(a) shows the utility gained ( $y$-axes) by norm supporting agents, its $x$-axes shows the enforcer to meek agent ratio. Figure 6(b) instead shows the utility gained by norm violating agents. In both figures each line represents the amount of violating agents in the system. As the number of enforcers increases there is a tendency for norm supporters to gain more utility, while the opposite tendency is observed for violator agents. When the number of enforcer agents is low, the utility gained by violator agents is much higher than the one gained by norm supporters. As the number of enforcer agents grows the roles are reversed. The inflection point depends on the amount of violator agents in the system. For simulations with $10 \%$ of violator agents, supporting the norm becomes rational when the enforcer to meek ratio is higher than 1.25. For simulations with $50 \%$ of violator agents, the ratio needs to be higher than 0.7 . The rest of simulations have inflection points between those two values.

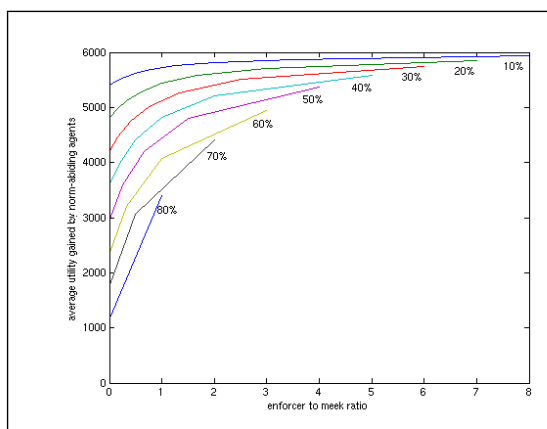

(a) norm-abiding agents

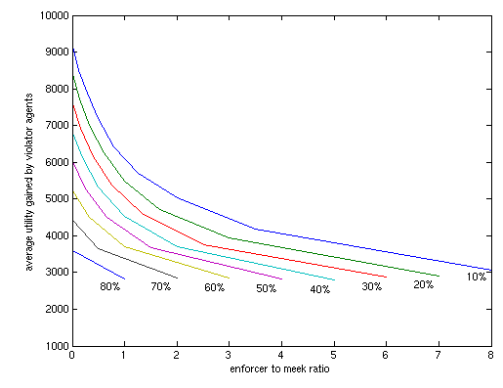

(b) norm-violating agents

Fig. 6. Utility gained by norm-abiding

It is interesting to note that even though meek agents receive more violations as the number of enforcer agents grows (see Figure 7(a)), the utility gained by them surprisingly increases (see Figure 7(b)). This is due to the fact that meek agents are still able to interact with other norm-abiding agents. Since violators are being blocked the ratio of defection to cooperation is lowered and the utility is increased.

\section{Future Work}

This paper is part of ongoing research on norm enforcement. Future work will relax the set of assumptions about agents, by giving them the ability to change 


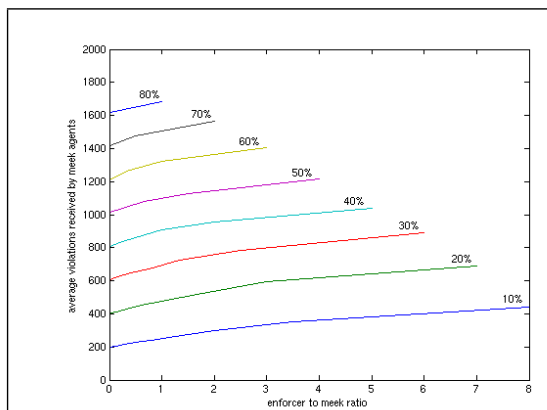

(a) Violations Received

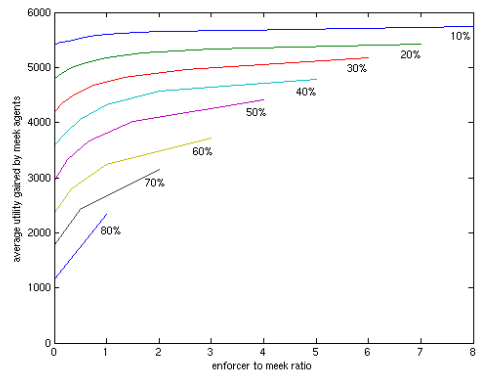

(b) Utility gained

Fig. 7. Local blocking rule increases both utility and violations to meek agents

their strategies in time, to lie, and to allow enforcer agents to violate the norm (i.e., corrupt enforcers). The assumption of perfect information will be relaxed by adding uncertainty and noise. For these cases elaborate gossip techniques and reputation management will allow agents to enforce the norm. In future work the agent's reputation will be modeled not through gossip but through interaction overhearing. Mediating agents could overhear the interactions instead of waiting for interacting agents to report the outcome. More so, other conservative blocking strategies can be studied; such as blocking off agents that mediate norm violators, or blocking agents until they are shown to be norm-abiders.

Furthermore, the impact of other network parameters and dynamic networks will be analyzed. New links between agents could be added dynamically and test how this affects norm enforcement. New enforcement techniques will be studied to take advantage of dynamic networks.

Finally, other studies have shown that the efficiency of enforcement diminishes when enforcement conveys a cost to the enforcing agent $[1,8]$. In future work there will be cost associated to blockage. One way to associate cost to enforcers is by removing their ability to stop agents from interacting with them. In this case, enforcers can withhold information from known violators, but if asked will have to interact with them and endure the norm violation.

\section{Acknowledgments}

This work is supported by the FP6 OpenKnowledge ${ }^{4}$ Project. A. Perreau de Pinninck is supported by a CSIC predoctoral fellowship under the I3P program, and M. Schorlemmer is supported by a Ramón y Cajal research fellowship from Spain's Ministry of Education and Science, both of which are partially funded by the European Social Fund.

\footnotetext{
${ }^{4}$ http://www.openk.org
} 


\section{References}

1. Robert Axelrod. An evolutionary approach to norms. The American Political Science Review, 80:1095-1111, 1986.

2. Guido Boella and Leendert van der Torre. Enforceable social laws. In AAMAS '05: Proceedings of the Fourth International Joint Conference on Autonomous Agents and Multiagent Systems, pages 682-689, 2005.

3. Jeffrey Carpenter, Peter Matthews, and Okomboli Ong'ong'a. Why punish: Social reciprocity and the enforcement of prosocial norms. Journal of Evolutionary Economics, 14(4):407-429, 2004.

4. Cristiano Castelfranchi, Rosaria Conte, and Mario Paoluccci. Normative reputation and the costs of compliance. Journal of Artificial Societies and Social Simulation, 1(3), 1998.

5. Jordi Delgado. Emergence of social conventions in complex networks. Artificial Intelligence, 141(1):171-185, 2002.

6. Amandine Grizard, Laurent Vercouter, Tiberiu Stratulat, and Guillaume Muller. A peer-to-peer normative system to achieve social order. In AAMAS '06 Workshop on Coordination, Organization, Institutions and Norms in agent systems (COIN), 2006.

7. David Hales. Group reputation supports beneficent norms. Journal of Artificial Societies and Social Simulation, 5(4), 2002.

8. Douglas D. Heckathorn. Collective sanctions and compliance norms: a formal theory of group-mediated social control. American Sociological Review, 55(3):366384, 1990.

9. James E. Kittock. The impact of locality and authority on emergent conventions: initial observations. In AAAI'94: Proceedings of the Twelfth National Conference on Artificial Intelligence, volume 1, pages 420-425, Menlo Park, CA, USA, 1994. American Association for Artificial Intelligence.

10. Josep M. Pujol, Jordi Delgado, Ramon Sangüesa, and Andreas Flache. The role of clustering on the emergence of efficient social conventions. In IJCAI '05: Proceedings of the Nineteenth International Joint Conference on Artificial Intelligence, pages 965-970, 2005.

11. Michael Taylor. Community, Anarchy \& Liberty. Cambridge University Press, 1982.

12. Adam Walker and Michael Wooldridge. Understanding the emergence of conventions in multi-agent systems. In Victor Lesser, editor, Proceedings of the First International Conference on Multi-Agent Systems, pages 384-389, San Francisco, CA, 1995. MIT Press.

13. Duncan J. Watts and Steven H. Strogatz. Collective dynamics of small-world networks. Nature, (393):440-442, 1998.

14. Fabiola López y López, Michael Luck, and Mark d'Inverno. Constraining autonomy through norms. In $A A M A S$ '02: Proceedings of the First International Joint Conference on Autonomous Agents and Multiagent Systems, pages 674-681, New York, NY, USA, 2002. ACM Press.

15. Stephen Younger. Reciprocity, sanctions, and the development of mutual obligation in egalitarian societies. Journal of Artificial Societies and Social Simulation, 8(2), 2005. 